\title{
Anomalous Metapopulation Dynamics on Scale-Free Networks
}

DOI:

10.1103/PhysRevLett.118.098301

\section{Document Version}

Final published version

Link to publication record in Manchester Research Explorer

\section{Citation for published version (APA):}

Fedotov, S., \& Stage, H. (2017). Anomalous Metapopulation Dynamics on Scale-Free Networks. Physical Review Letters, 118, [098301]. https://doi.org/10.1103/PhysRevLett.118.098301

\section{Published in:}

Physical Review Letters

\section{Citing this paper}

Please note that where the full-text provided on Manchester Research Explorer is the Author Accepted Manuscript or Proof version this may differ from the final Published version. If citing, it is advised that you check and use the publisher's definitive version.

\section{General rights}

Copyright and moral rights for the publications made accessible in the Research Explorer are retained by the authors and/or other copyright owners and it is a condition of accessing publications that users recognise and abide by the legal requirements associated with these rights.

\section{Takedown policy}

If you believe that this document breaches copyright please refer to the University of Manchester's Takedown Procedures [http://man.ac.uk/04Y6Bo] or contact uml.scholarlycommunications@manchester.ac.uk providing relevant details, so we can investigate your claim.

\section{OPEN ACCESS}




\title{
Anomalous Metapopulation Dynamics on Scale-Free Networks
}

\author{
Sergei Fedotov ${ }^{*}$ and Helena Stage ${ }^{\dagger}$ \\ School of Mathematics, The University of Manchester, Manchester M13 9PL, United Kingdom
}

(Received 14 October 2016; published 3 March 2017)

\begin{abstract}
We model transport of individuals across a heterogeneous scale-free network where a few weakly connected nodes exhibit heavy-tailed residence times. Using the empirical law of the axiom of cumulative inertia and fractional analysis, we show that "anomalous cumulative inertia" overpowers highly connected nodes in attracting network individuals. This fundamentally challenges the classical result that individuals tend to accumulate in high-order nodes. The derived residence time distribution has a nontrivial $U$ shape which we encounter empirically across human residence and employment times.
\end{abstract}

DOI: 10.1103/PhysRevLett.118.098301

Introduction.-In the past few decades, many metapopulation models have been developed describing reactiontransport processes on scale-free networks [1-10]. The idea that the overall population can be understood as a series of spatially connected but separated "patches" [11] is useful in many areas, including the migration of humans between cities [12], scientific collaborations [13], the spread of epidemic diseases via individual movement $[4,8,9,14]$, and international air travel [15]. Often networks are assumed to be scale free such that the order (number of connections) of each node (patch) is drawn from a power-law distribution, $P(k) \sim k^{-\gamma}, \gamma>0$ [16-19].

While considerations of stochastic movement of individuals on a complex network are very challenging, much progress has been made using a mean-field approximation across nodes of equal order. One introduces the mean number of individuals $N_{k}(t)=1 / \eta_{k} \sum_{i} \rho_{i, k}(t)$, where $\rho_{i, k}(t)$ is the number of individuals in the $i^{\text {th }}$ node of order $k$ and $\eta_{k}$ is the number of nodes of order $k$ [3-5,7]. The equation describing transport between nodes can be written as

$$
\frac{\partial N_{k}}{\partial t}=-\rrbracket_{k}(t)+k \sum_{k^{\prime}} P\left(k^{\prime} \mid k\right) \frac{\mathbb{k}_{k^{\prime}}(t)}{k^{\prime}},
$$

where $\mathbb{1}_{k}(t)$ is the flux out of a node (patch) of order $k$ and $P\left(k^{\prime} \mid k\right)$ is the conditional probability that a link exists from a node of order $k$ to a node of order $k^{\prime}[3,9,18]$. Commonly, it is assumed that the residence time in a node (before moving elsewhere) is exponentially distributed and independent of degree $k$ [3-6]. This implies a constant escape rate $\lambda$ for which the flux is

$$
\rrbracket_{k}(t)=\lambda N_{k}(t)
$$

$[6,7,20]$. The assumption of an uncorrelated network such that $P\left(k^{\prime} \mid k\right)=k^{\prime} P\left(k^{\prime}\right) /\langle k\rangle[18,21,22]$, together with Eq. (2), leads to the well-known steady-state result $[3,4]$

$$
N_{k}^{s}=\frac{k}{\langle k\rangle} \sum_{k^{\prime}} P\left(k^{\prime}\right) N_{k^{\prime}}^{s}=\frac{k}{\langle k\rangle}\left\langle N^{s}\right\rangle .
$$

It follows from Eq. (3) that the mean number of individuals in a node (patch) increases with the order. One can interpret this as individuals spending more time in well-connected nodes. This famous result has been key in developing, e.g., the PageRank algorithm and is still fundamental in our intuition regarding network behavior. However, such conclusions are heavily based on the assumption that the movement between patches can be approximated by a Poisson process. That is, the interval between consecutive escapes from a node (residence time) follows an exponential probability density function (PDF), $\psi(\tau)=\lambda e^{-\lambda \tau}$. New work has emerged in recent years indicating that human activity is not Poisson distributed [23]. In particular, the efforts of Barabási and others have demonstrated that human activity often involves heavy-tailed or Pareto type PDFs [19,24-30]. This is particularly relevant for human mobility due to the empirical sociological law known as "the axiom of cumulative inertia" (ACI), which suggests that the probability of a person remaining in a state increases with the associated residence time $[31,32]$. The ACI can be reformulated in terms of a power-law residence time [33] with PDF,

$$
\psi(\tau)=\frac{\mu}{\tau+\tau_{0}}\left(\frac{\tau_{0}}{\tau+\tau_{0}}\right)^{\mu},
$$

for fixed constants $\mu, \tau_{0}>0$. For the anomalous case, $\mu<1$, instead of Eq. (2), we obtain a fractional flux $\square_{k}^{a}(t)$ out of a patch

$$
\square_{k}^{a}(t)=\frac{1}{\Gamma(1-\mu) \tau_{0}^{\mu}}{ }_{0} \mathcal{D}^{1-\mu} N_{k}(t),
$$

where ${ }_{0} \mathcal{D}^{1-\mu}$ is the Riemann-Liouville fractional derivative defined as

$$
{ }_{0} \mathcal{D}^{1-\mu} N_{k}(t)=\frac{1}{\Gamma(\mu)} \frac{d}{d t} \int_{0}^{t} \frac{N_{k}(\tau)}{(t-\tau)^{1-\mu}} d \tau
$$

[34-37] (details in Supplemental Material [38] and following subsection). To the authors' knowledge, no work has yet been 
done investigating the effect of anomalous fluxes like Eq. (5) on Eq. (3) and the subsequent implications for the long-time distribution of network individuals.

So what happens if we introduce an anomalous flux like Eq. (5) into heterogenenous network models? Surprisingly, in the case of $\mu<1$, Eq. (3) was radically altered beyond the effects attributable to small perturbations. Accumulation in high-order nodes did occur, but as a short-lived transient state of the network. In the long-time limit, individuals aggregated in the patches with power-law residence times, invalidating Eq. (3). This fundamentally challenges the classically held belief that individuals will tend to accumulate in the nodes of highest order [3,4,6-9]. Furthermore, these aggregated individuals exhibit a nontrivial $U$-shaped residence time distribution, which we find to be ubiquitous across social phenomena of mobility and employment. In what follows, we develop an anomalous metapopulation model describing this behavior.

Anomalous nodes in a network.-We concern ourselves with transport on a heterogeneous scale-free network containing some nodes with power-law distributed residence times [see Eq. (4)] and the rest with exponentially distributed residence times. We call nodes "anomalous" if their average residence time, $\langle T\rangle=\int_{0}^{\infty} \tau \psi(\tau) d \tau$, diverges. This occurs when $\mu<1$ and is the case we shall focus on (empirical evidence for its existence to follow). We intend to show that even in the extreme case of few connections, these power-law nodes are dominant in attracting network individuals. Individuals leave nodes with rates $\mathbb{T}$. For exponential residence times, $\mathbb{T}$ is constant and Eq. (2) describes the flux. Else for power-law residence times, $\mathbb{T}(\tau)=\mu /\left(\tau+\tau_{0}\right)$ yields Eq. (4) [33], using $\psi(\tau)=\mathbb{T}(\tau) \exp \left[-\int_{0}^{\tau} \mathbb{T}(u) d u\right]$. The inverse residence time dependence of $\mathbb{T}(\tau)$ is another manifestation of the ACI, which we motivate as follows. Consider a person moving to a new city: over time, they develop a social circle, gain steady employment, or enter family life. Consequently, the longer their residence time, the more settled they become and are thus less likely to leave $[39,40]$.

For power-law residence times, it is convenient to consider the renewal measure $h(t)$. This function can be understood as the number of events per unit time, where an "event" is an individual leaving a node. $h(t)$ obeys the renewal equation $h(t)=\psi(t)+\int_{0}^{t} h(\tau) \psi(t-\tau) d \tau$ [41]. One can rewrite the flux $\rrbracket_{k}(t)$ from Eq. (1) as

$$
\mathbb{\square}_{k}(t)=\frac{d}{d t} \int_{0}^{t} h(t-\tau) N_{k}(\tau) d \tau,
$$

which is valid for all $\psi(\tau)$ (see [35], Ch. 5 for the derivation). Clearly, for constant $h(t)=\lambda$, we obtain Eq. (2). The case $\mu<1$ in Eq. (4) requires a fractional analysis of the renewal measure such that we obtain

$$
h(t)=\frac{t^{-1+\mu}}{\Gamma(1-\mu) \Gamma(\mu) \tau_{0}^{\mu}}
$$

as $t \rightarrow \infty$ [35,42]. Substituting Eq. (8) into Eq. (7) corresponds to the anomalous fractional flux $\square_{k}^{a}(t)$ of Eq. (5). We will show that this flux changes the preferential residence of individuals in well-connected nodes in favor of those with anomalous flux, even if these are weakly connected. This corresponds to the dominance of low-order nodes (patches) with flux $\square_{k}^{a}(t)$ over high-order nodes with flux $\mathbb{\square}_{k}=\lambda N_{k}(t)$. Let us for simplicity, assume only anomalous nodes to have order $k_{a} \ll\langle k\rangle$ (nodes are weakly connected). The flux $\llbracket(t)$ from the balance Eq. (1) becomes

$$
\mathbb{\square}_{k}(t)=\left[1-\delta_{k k_{a}}\right] \lambda N_{k}(t)+\delta_{k k_{a}} \square_{k}^{a}(t),
$$

where $\delta_{k k_{a}}$ is the discrete Kronecker delta. By analysis of Eq. (1) (details in Supplemental Material [38]), it follows that in the limit $t \rightarrow \infty$,

$$
N_{k}(t) \eta_{k} \rightarrow \delta_{k k_{a}} N
$$

where $N$ is the total number of individuals in the network and $\eta_{k}$ the number of nodes with order $k$. Hence, the anomalous nodes jointly contain all individuals as $t \rightarrow \infty$. This key result contrasts with the popular belief that wellconnected nodes are more attractive. Furthermore, similar results cannot be replicated by naively introducing nodes with very low escape rates, $\lambda \ll 1$.

We confirm the result of Eq. (10) by Monte Carlo simulations illustrated in Fig. 1. A scale-free $\left[P(k) \sim k^{-\gamma}\right.$, $\gamma=1.5,2.5]$, uncorrelated network was constructed using the Molloy-Reed algorithm, containing $\eta_{k_{a}}=3$ anomalous nodes of order $k_{a}=4$ [43]. This was compared with another network where all nodes have exponential

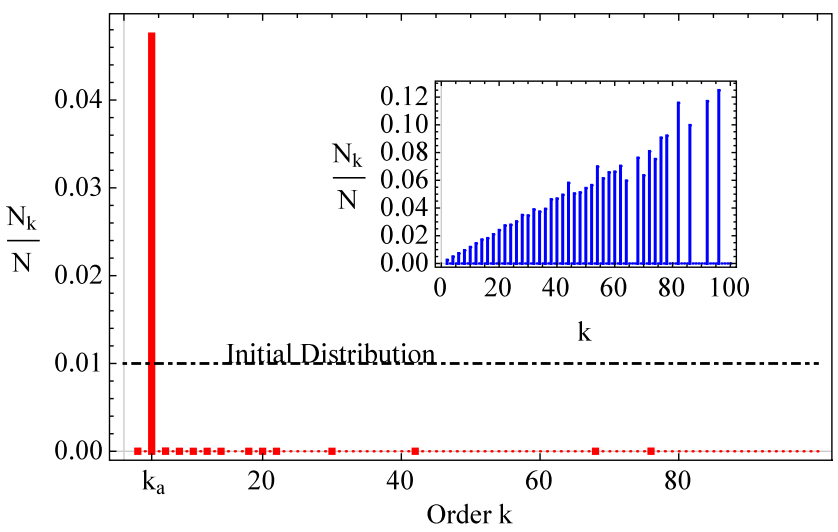

FIG. 1. $\quad N_{k}(t) / N$ for a network of 100 nodes with 3 anomalous nodes, all of order $k_{a}=4$, with $\mu=0.5, \tau_{0}=1$, and $N=10^{5}$ individuals (initially distributed uniformly). Individuals eventually aggregate in the anomalous nodes. The inset shows $N_{k} / N$ if all nodes have constant escape rates $\mathbb{T}=\lambda=2$, equivalent to networks with $T(\tau)$ for $\tau_{0}=1, \mu=3$. 
residence times and flux $\rrbracket_{k}(t)=\lambda N_{k}(t)$. Both simulations were carried out with 100 nodes and $N=10^{5}$ individuals. Simulations were also done for networks with up to $10^{4}$ nodes with qualitatively similar results but a longer transient state. Simulations almost immediately showed the individuals accumulating in nodes according to their order as described by Eq. (3). However, this behavior was transient as the individuals then slowly moved into the anomalous nodes. We observed an initially fast rate of organization into the classically expected configuration which then, with a (power-law) slow rate, changed into a preference for the anomalous nodes. This leads to the peak in $N_{k}(t)$ at $k=k_{a}$. One can allow nonanomalous nodes of order $k_{a}$ in the network, though these will gradually be emptied. The only consequence is a reduced value of $N_{k_{a}}(t)$ as $\eta_{k_{a}}$ grows. Similarly, our findings are qualitatively unchanged for any $k_{a}>0$; this only changes how quickly accumulation occurs.

PDFs for residence times like Eq. (4) have previously been applied to random walks [44-46]. However, these papers do not consider the effects on a network structure nor the details pertaining to the accumulated individuals. Related work exists considering heavy-tailed residence times in biased Watts-Strogatz networks, which demonstrated pair aggregation akin to self-chemotacticlike forcing [36]. Other pattern formation on scale-free networks has been observed with order-dependent escape rates [47]. Patterns or dominant behaviors are known to arise in networks, either as a result of heterogeneities in $P(k)$ [20] and the role of extreme values of $k$ [48] or following the interplay of these with escape rates or node reaction dynamics [5].

Two-state system.-From our simulations, we observe the formation of two states in the network. There is a slow transport of individuals to the anomalous patches, arising from the gradual depletion of the surrounding nodes. Consequently, we can regard this peak in individuals as one state $\mathbb{S}_{1}$ and the remainder of the nodes as the other state $\mathbb{S}_{2}$. This picture (see Fig. 2) allows us to find the rate at which the aforementioned peak grows. The corresponding equations to Eq. (1) are

$$
\frac{d N_{1}}{d t}=\mathbb{\square}_{2}(t)-\square_{1}(t), \quad N_{2}(t)=N-N_{1}(t),
$$

where $N_{i}(t), \mathbb{\nabla}_{i}(t)$ are the respective mean number of individuals in, and flux from, state $\mathbb{S}_{i}$. Hence, the fluxes $\mathbb{S}_{2} \leftrightarrow \mathbb{S}_{1}$ in analogy to Eqs. (2) and (7) are given by $\mathbb{1}_{2}(t)=$ $\lambda N_{2}(t)$ and $\square_{1}(t)=d / d t \int_{0}^{t} h(t-\tau) N_{1}(\tau) d \tau$, where $h(t)$ follows Eq. (8). In the limit of $t \rightarrow \infty$, we neglect the derivative $d N_{1} / d t \approx 0$ such that Eq. (11) becomes

$$
N=N_{1}(t)+\frac{1}{\lambda} \frac{d}{d t} \int_{0}^{t} h(t-\tau) N_{1}(\tau) d \tau .
$$

This evaluates to

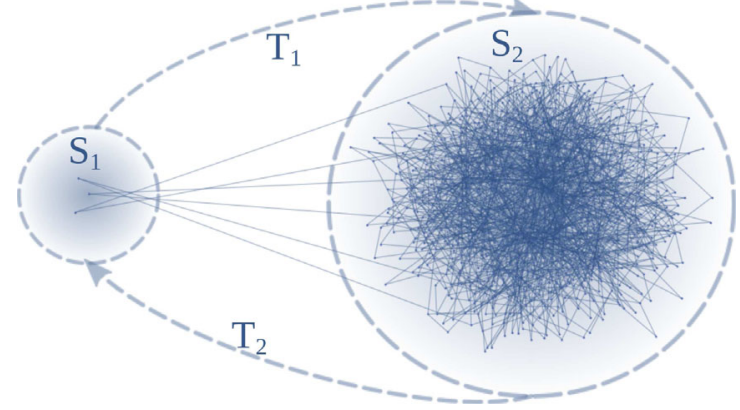

FIG. 2. Network separation into two states 1,2 with transition rates $\mathbb{T}_{1}, \mathbb{T}_{2}$. The exact number of nodes in each state and the number of connections between the states is insignificant so long as $\mathbb{S}_{2}$ contains the majority of nodes. The intention is to demonstrate the attractiveness of $\mathbb{S}_{1}$, even in the extreme case where there are very few connections.

$N_{1}(t)=N\left(1-\frac{h(t)}{\lambda}\right) \rightarrow N, \quad N_{2}(t)=\frac{N h(t)}{\lambda} \rightarrow 0$

as $t \rightarrow \infty$. Equation (13) thus describes the power-law slow nonstationary aggregation, which is consistent with Eq. (10). This phenomenon has been observed previously in other contexts [49], though its implications for networks has hitherto not been considered. Internal connections in $\mathbb{S}_{2}$ are negligible as they simply contribute slightly to the probability of remaining in $\mathbb{S}_{2}$ (thus increasing the time taken to aggregate in $\mathbb{S}_{1}$ but not the overall behavior).

Using the same parameters, Monte Carlo simulations of the whole network were carried out to test the prediction of Eq. (13) and the validity of the two-state simplification. As shown in the inset of Fig. 3, the simulation is in agreement with theoretical expectations and converges to Eq. (13) as $t \rightarrow \infty$. The suitability of the fit thus supports our two-state simplifying assumption. Note that even at large times, oscillations occur around the maximum, indicating that an equilibrium state does not exist.

Preferential Residence.-The aim now is to provide empirical evidence for the anomalous attractiveness of nodes with power-law residence time PDFs like Eq. (4), with $\mu<1$. Equation (13) and Fig. 1 show that individuals will tend to reside in $\mathbb{S}_{1}$, but what is the fine structure of these residence times? We separate the number of individuals according to their residence times. Hence, $n_{1}(t, \tau) \Delta \tau$ gives the number of individuals with residence times in the interval $(\tau, \tau+\Delta \tau)$, with initial condition $n_{1}(0, \tau)=n_{1}^{0} \delta(\tau)$, where $n_{1}^{0} \ll N$. Consequently, $N_{1}(t)=\int_{0}^{t} n_{1}(t, \tau) d \tau$. We can write $n_{1}$ in terms of the renewal measure $h(t)$ [41]

$$
n_{1}(t, \tau)=N h(t-\tau) \Psi(\tau),
$$

where the survival function, $\Psi(\tau)=\int_{\tau}^{\infty} \psi(u) d u=$ $\left(\tau_{0} / \tau+\tau_{0}\right)^{\mu}$, follows from Eq. (4). Substituting Eq. (8) and letting $t \rightarrow \infty$, we find a $U$-shaped distribution 


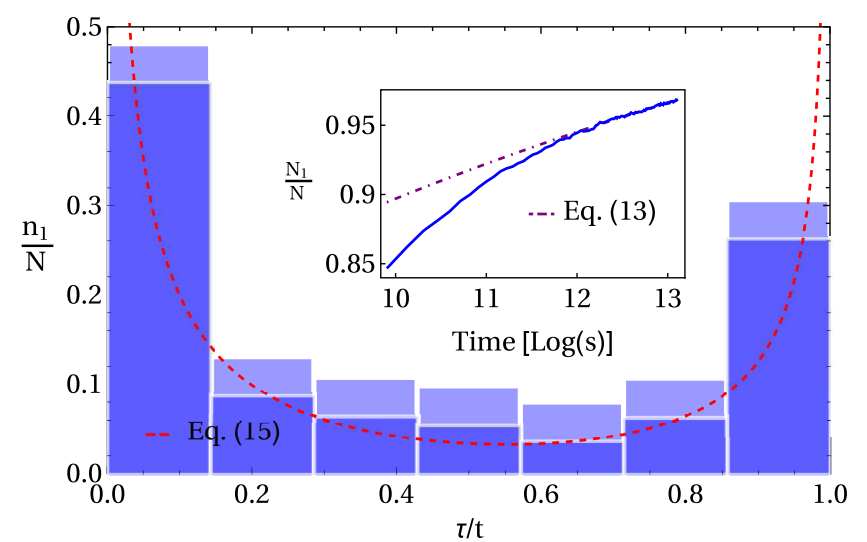

FIG. 3. The histogram shows $n_{1}(t, \tau) / N$, sampled from 12288 households in Milwaukee from 1950-1962. There is reasonable agreement between the data and Eq. (15) for $\mathbb{T}(\tau) \approx$ $0.55 /(0.22+\tau)$ at $t=12$ years between $1950-1962$. Estimated errors are indicated by the shaded regions. The inset shows $N_{1}(t) / N$ as measured from our simulations (using same parameters as Fig. 1), thus illustrating the aggregation of individuals in $\mathbb{S}_{1}$ as described by Eq. (13).

$$
n_{1}(t, \tau) \simeq \frac{N}{\Gamma(1-\mu) \Gamma(\mu) \tau^{\mu}(t-\tau)^{1-\mu}}
$$

This result is consistent with the generalized arc sine distributions for backward recurrence times [50] (see p. 445 where $x=\tau / t$ ), which only holds for $\mu<1$.

We now compare Eq. (15) with empirical observations. By analyzing data from an objective housing survey carried out among 16000 households in Milwaukee between 1950-1962, we obtained the residence times since moving into the current home [51]. This was done over an interval of 12 years and allows us to "track" households and their moves as illustrated in Fig. 3. The key features of the plot are the peaks in $n_{1} / N$ at $\tau \ll t$ and $\tau \sim t$, corresponding to the most likely residence times being very short or constituting the majority of the time. The same behavior is produced by Eq. (15) and is qualitatively very different from the predictions for $\mu>1$. In the latter case where the mean residence time $\langle T\rangle$ exists, one obtains the asymptotic result $n_{1}(t, \tau) \rightarrow \Psi(\tau) /\langle T\rangle$ [41]. This is a decaying function of residence time $\tau$ and does not provide a good description of the data in Fig. 3.

The presence of peaks at both low and high residence times in our data is consistent with the axiom of cumulative inertia in that most of the individuals will either be longterm residents (which do not move) or the sum of the continued in- or outflux of new arrivals. We stress that these peaks only arise if $\mu<1$ is also satisfied. Our findings are consistent with similar data obtained by the Bureau of Census during the American Housing Surveys in the period 1985-1993 [39]. Inspired by the results for human residence, the authors carried out a survey amongst permanently employed academics at The University of
Manchester, and found a $U$-shaped distribution of employment times like Fig. 3 (see Supplemental Material [38]). We refer to the former case as "academic trapping": once a permanent position at a research institution has been found, the dynamics follow the ACI. Convergence rates to Eq. (15) are discussed in [52].

Our assumption that individuals follow the ACI in some nodes and not in others is used purely for the sake of simplification. To justify this, we assume now all nodes follow the ACI as given by Eq. (4) such that some nodes are anomalous with $\mu<1$ and others have $\mu>1$. That is, all network dynamics are non-Markovian with fluxes

$\square_{k}(t)=\left[1-\delta_{k k_{a}}\right] \frac{d}{d t} \int_{0}^{t} h(t-u) N_{k}(u) d u+\delta_{k k_{a}} \square_{k}^{a}(t)$,

where $\square_{k}^{a}$ is the fractional flux defined by Eq. (5) and $h(t)$ the renewal measure for nodes with $\mu>1$. Numerical simulations of this network qualitatively mimic Fig. 1, with aggregation in the nodes with $\mu<1$. This is understood via the mean residence time $\langle T\rangle$ of the nonanomalous nodes. When $\mathbb{T}_{k}(\tau)=\lambda$, one finds that $\langle T\rangle \approx 1 / \lambda$. Else, when $\mathbb{T}_{k}(\tau)=\mu_{k} /\left(\tau+\tau_{0}\right)$ and $\mu_{k}>1$, one obtains $\langle T\rangle=\tau_{0} /\left(\mu_{k}-1\right)$. Hence, despite one treatment being Markovian and the other non-Markovian, both escape rates lead to finite amounts of time spent in the nodes. Recalling that when $\mu<1,\langle T\rangle \rightarrow \infty$, it becomes clear why the anomalous nodes dominate the aggregation. The residence time dependence, inspired by the ACI, is alone insufficient to change the qualitative behavior of the network.

Discussion and Conclusion.-It is a commonly held belief that individuals in a scale-free network will prefer highly connected nodes (patches). Our work fundamentally challenges this notion when individuals' flux follows the anomalous axiom of cumulative inertia as described by Eq. (5). We have shown both analytically and numerically that the flux out of anomalous nodes with power-law residence times outperforms highly connected nodes in the aggregation of network individuals. We further provide empirical evidence for the associated residence time distribution $n(t, \tau)$ of aggregated individuals, motivated by the ACI [40]. Our findings constitute an important result in the context of network theory given the wealth of evidence that human behavior, such as our habits on web surfing and with television, follows heavy-tailed distributions [28-30]. Other examples of such distributions include messaging, queuing, and prioritizing tasks [25,27,53].

Empirical data suggest that human residence and academic employment fall into the case of anomalous behavior. Long durations of permanent employment lead to "academic trapping" where dynamics obey the ACI. Despite our analysis only being valid in cases $\mu<1$, we demonstrate empirically that this is a ubiquitous example in population movement, with variations arising depending on the nature of residence (renting or owning a home). 
Strikingly, it is the fractional analysis of node dynamics which uncovers the essential features of our model: anomalous accumulation and a nontrivial $U$-shaped residence time distribution. Our findings need not apply only to residence times in geographical regions or employed positions, but could equally describe entrenchment of ideological beliefs, convictions, etc.

We believe our findings will have a significant impact on network metapopulation models studying epidemiology (e.g. the Susceptible-Infected-Recovered (SIR) model) $[4,8,9,14]$. It is well known that the time spent by travelers at a destination is characterized by wide fluctuations, which crucially affects the chance and duration of mixing events and therefore, has a strong impact on the spread of an emerging disease [54]. We thus expect anomalous patches to be significant in understanding how diseases might spread when individuals are reluctant to leave an area. Some work on memory effects including residence time dependence [54,55], second-order Markov processes [56], and the effects of individual movements [57] has already been carried out.

The authors would like to thank H. Berry, S. Falconer, N. Korabel and P. Straka for useful discussions. This work is supported by EPSRC Grant No. EP/N018060/1.

*sergei.fedotov@manchester.ac.uk

†helena.stage@manchester.ac.uk

[1] C. J. Rhodes and R. M. Anderson, Nature (London) 381, 600 (1996).

[2] Grenfell and Bolker, Ecol. Lett. 1, 63 (1998).

[3] V. Colizza, R. Pastor-Satorras, and A. Vespignani, Nat. Phys. 3, 276 (2007).

[4] V. Colizza and A. Vespignani, Phys. Rev. Lett. 99, 148701 (2007).

[5] V. Colizza and A. Vespignani, J. Theor. Biol. 251, 450 (2008).

[6] D. Balcan and A. Vespignani, Nat. Phys. 7, 581 (2011).

[7] A. Vespignani, Nat. Phys. 8, 32 (2012).

[8] M. Tizzoni, K. Sun, D. Benusiglio, M. Karsai, and N. Perra, Sci. Rep. 5, 15111 (2015).

[9] G. E. Leventhal, A. L. Hill, M. A. Nowak, and S. Bonhoeffer, Nat. Commun. 6, 6101 (2015).

[10] J. Ripoll, A. Avinyó, M. Pellicer, and J. Saldaña, Phys. Rev. E 92, 022809 (2015).

[11] I. Hanski, Nature (London) 396, 41 (1998).

[12] S. Riley, Science 316, 1298 (2007).

[13] A.-L. Barabási, H. Jeong, Z. Néda, E. Ravasz, A. Schubert, and T. Vicsek, Physica A (Amsterdam) 311, 590 (2002).

[14] T. D. Hollingsworth, N. M. Ferguson, and R. M. Anderson, Nat. Med. 12, 497 (2006).

[15] R. Guimerà, S. Mossa, A. Turtschi, and L. A. N. Amaral, Proc. Natl. Acad. Sci. U.S.A. 102, 7794 (2005).

[16] M. Newman, A.-L. Barabási, and D. J. Watts, The Structure and Dynamics of Networks (Princeton University Press, Princeton and Oxford, 2011).

[17] A.-L. Barabási and R. Albert, Science 286, 509 (1999).
[18] R. Pastor-Satorras, A. Vázquez, and A. Vespignani, Phys. Rev. Lett. 87, 258701 (2001).

[19] A.-L. Barabási, Science 325, 412 (2009).

[20] H. Yang, M. Tang, and T. Gross, Sci. Rep. 5, 13122 (2015).

[21] M. E. J. Newman, S. H. Strogatz, and D. J. Watts, Phys. Rev. E 64, 026118 (2001).

[22] A. Barrat, M. Barthélemy, and A. Vespignani, Dynamical Processes on Complex Networks (Cambridge University Press, Cambridge, 2008).

[23] L. M. A. Bettencourt, J. Lobo, D. Helbing, C. Kühnert, and G. B. West, Proc. Natl. Acad. Sci. U.S.A. 104, 7301 (2007).

[24] A.-L. Barabási, Nature (London) 435, 207 (2005).

[25] A. Vázquez, Phys. Rev. Lett. 95, 248701 (2005).

[26] D. Brockmann, L. Hufnagel, and T. Geisel, Nature (London) 439, 462 (2006).

[27] A. Vázquez, J. G. Oliveira, Z. Dezsö, K.-I. Goh, I. Kondor, and A.-L. Barabási, Phys. Rev. E 73, 036127 (2006).

[28] A. Vazquez, B. Rácz, A. Lukács, and A.-L. Barabási, Phys. Rev. Lett. 98, 158702 (2007).

[29] C. Liu, R. W. White, and S. Dumais, in Proceedings of the 33rd International ACM SIGIR Conference on Research and Development in Information Retrieval (ACM, New York, 2010), pp. 379-386.

[30] Y. Li, Y. Zhang, and R. Yuan, in 2012 IEEE International Conference on Communications (IEEE, New York, 2012), pp. 2093-2097.

[31] G. C. Myers, R. McGinnis, and G. Masnick, Eugen. Q. 14, 121 (1967).

[32] R. McGinnis, Am. Sociol. Rev 33, 712 (1968).

[33] B. Singer and S. Spilerman, Sociol. Methodol. 5, 356 (1974).

[34] K. S. Miller and B. Ross, An Introduction to the Fractional Calculus and Fractional Differential Equations (WileyBlackwell, New York, 1993).

[35] J. Klafter and I. M. Sokolov, First Steps in Random Walks: From Tools to Applications (Oxford University Press, Oxford, 2011).

[36] C. N. Angstmann, I. C. Donnelly, B. I. Henry, and T. A. M. Langlands, Phys. Rev. E 88, 022811 (2013).

[37] C. N. Angstmann, I. C. Donnelly, B. I. Henry, and T. A. M. Langlands, Math. Model. Nat. Phenom. 11, 142 (2016).

[38] See Supplemental Material at http://link.aps.org/ supplemental/10.1103/PhysRevLett.118.098301 for derivation details and further information.

[39] S. Anily, J. Hornik, and M. Israeli, J. Bus. Econ. Stat. 17, 373 (1999).

[40] P. S. Morrison and W. A. V. Clark, CCPR Working Paper Series (CCPR, Los Angeles, 2015).

[41] D. R. Cox and H. D. Miller, The Theory of Stochastic Processes (CRC Press, Boca Raton, 1977).

[42] C. Godrèche and J. M. Luck, J. Stat. Phys. 104, 489 (2001).

[43] M. Molloy and B. Reed, Random Struct. Algorithms 6, 161 (1995).

[44] S. Fedotov and S. Falconer, Phys. Rev. E 85, 031132 (2012).

[45] S. Fedotov, Phys. Rev. E 88, 032104 (2013).

[46] S. Fedotov and N. Korabel, Phys. Rev. E 92, 062127 (2015).

[47] C. N. Angstmann, I. C. Donnelly, and B. I. Henry, Phys. Rev. E 87, 032804 (2013).

[48] G. Tanaka, K. Morino, and K. Aihara, Sci. Rep. 2, 232 (2012).

[49] A. I. Shushin, Phys. Rev. E 64, 051108 (2001). 
[50] W. Feller, An Introduction to Probability Theory and Its Applications (John Wiley \& Sons, Inc., New York, 1966), Vol. 2.

[51] W. A. V. Clark and J. O. Huff, Environ. Plann. A 9, 1357 (1977).

[52] H. Berry, T. Lepoutre, and Á. M. González, Acta Appl. Math. 145, 15 (2016).

[53] J. G. Oliveira and A.-L. Barabási, Nat. Commun. 437, 1251 (2005).
[54] C. Poletto, M. Tizzoni, and V. Colizza, J. Theor. Biol. 338, 41 (2013).

[55] C. Poletto, M. Tizzoni, and V. Colizza, Sci. Rep. 2, 476 (2012).

[56] M. Rosvall, A. V. Esquivel, A. Lancichinetti, J. D. West, and R. Lambiotte, Nat. Commun. 5, 4630 (2014).

[57] V. Belik, T. Geisel, and D. Brockmann, Phys. Rev. X 1, 011001 (2011). 